\title{
Teaching and implementing autonomous robotic lab walkthroughs in a biotech laboratory through model-based visual tracking.
}

\author{
Martin Wojtczyk ${ }^{1,2}$, Giorgio Panin ${ }^{1}$, Thorsten Röder ${ }^{1}$, Claus Lenz ${ }^{1}$, Suraj Nair ${ }^{1}$, Rüdiger \\ Heidemann ${ }^{2}$, Chetan Goudar ${ }^{2}$ and Alois Knoll ${ }^{1}$ \\ ${ }^{1}$ Informatics VI: Robotics and Embedded Systems, Technische Universität München, \\ Boltzmannstr. 3, D-85748 Garching b. München, Germany \\ ${ }^{2}$ Cell Culture Development, Global Biological Development, Bayer HealthCare LLC, \\ 800 Dwight Way, Berkeley, CA 94710, USA
}

\begin{abstract}
After utilizing robots for more than 30 years for classic industrial automation applications, service robots form a constantly increasing market, although the big breakthrough is still awaited. Our approach to service robots was driven by the idea of supporting lab personnel in a biotechnology laboratory. After initial development in Germany, a mobile robot platform extended with an industrial manipulator and the necessary sensors for indoor localization and object manipulation, has been evaluated by our group located in Berkeley, CA, USA. The determined goal of the mobile manipulator is to support the off-shift staff to carry out completely autonomous or guided, remote controlled lab walkthroughs, which we implement utilizing a recent development of our computer vision group: OpenTL - an integrated framework for model-based visual tracking.
\end{abstract}

Keywords: Service Robotics, Visual Tracking.

\section{INTRODUCTION}

A mobile robot, initially developed for sample management in a dedicated biotechnology pilot plant was successfully installed in a normal cell culture development laboratory for routine monitoring of multiple high cell density perfusion bioreactors. After demonstrating initial process robustness of 100 error-free and highly precise sampling cycles the project scope was extended towards versatility and usability of the mobile robot platform. ${ }^{1}$ Figure 1 shows the robot in the first, dedicated laboratory on site at Bayer HealthCare LLC to carry out a complete sample management process.

The robot consists of a battery driven, wheeled platform and a mounted industrial robot arm with a twofinger gripper attached to it. The proper interaction with the analysis and sample devices in the biotech lab is given by the use of a color camera for object recognition, a force/torque sensor to prevent damages and laser range finders at the front and the backside of the robot for localization. The on-board computer is responsible for localizing and navigating the platform with respect to the sensor data from the laser range finders. Furthermore the computer controls the mounted robot arm's movements. Precise device interactions with an accuracy of less than $1 \mathrm{~mm}$ are performed by pattern and object detection, utilizing the camera and dedicated computer vision algorithms in combination with realtime sensor feedback of a force-/torque sensor in between the robot's tool and its arm. ${ }^{2-5}$ Figure 2 presents an overview of the robot platform and the involved sensors and effectors.

Further author information: (Send correspondence to Martin Wojtczyk)

Martin Wojtczyk: E-mail: wojtczyk@in.tum.de, Telephone: +1-510-705-6810

Giorgio Panin: E-mail: panin@in.tum.de

Thorsten Röder: E-mail: roeder@in.tum.de

Claus Lenz: E-mail: lenz@in.tum.de

Suraj Nair: E-mail: nair@in.tum.de

Rüdiger Heidemann: E-mail: rudiger.heidemann.b@bayer.com

Chetan Goudar: E-mail: chetan.goudar.b@bayer.com

Alois Knoll: E-mail: knoll@in.tum.de, Telephone: +49-89-289-18104 


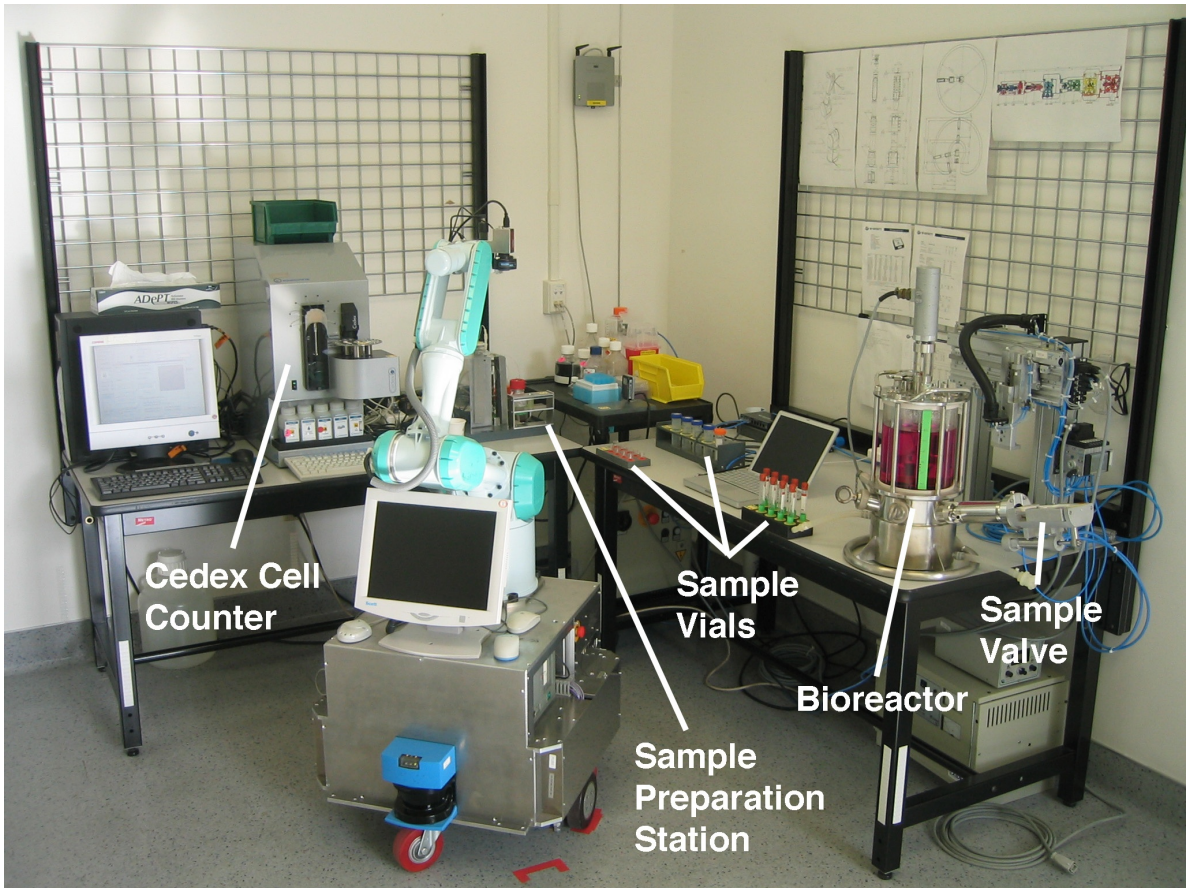

Figure 1. The mobile robot platform in its dedicated sample management laboratory for demonstration of process robustness.

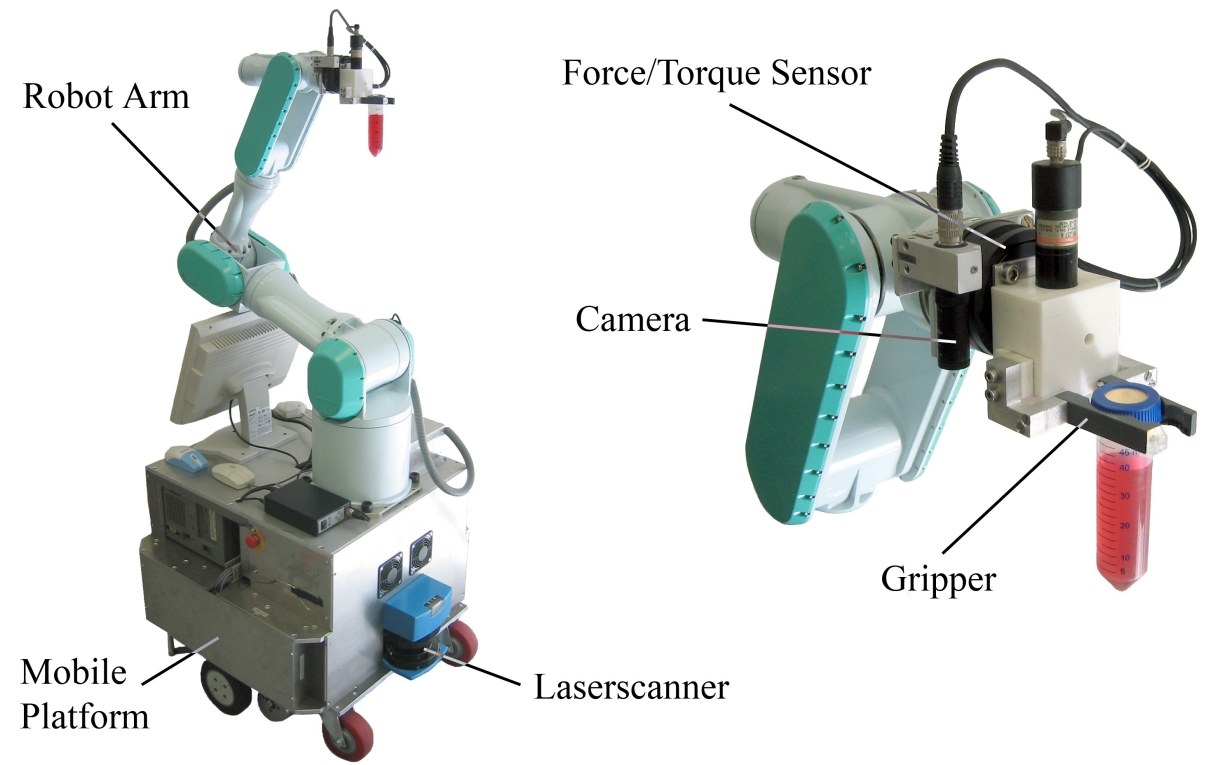

Figure 2. The mobile robot platform with its sensors and a close-up of its tool. 


\section{MOTIVATION}

The mobile robot platform has also been evaluated in a laboratory where it is sharing the lab space with human personnel. Within the extended project scope, the robot is monitoring high cell density perfusion reactors that typically operate for 3-6 months providing $24 / 7$ coverage, hence reduces the burden on off shift personnel.

The new environment however raises new challenges: modifications on the bioreactor stations which can occur on a daily basis, require an adaptive environment mapping and path planning, where the robot could use static mapping and path planning in the dedicated lab before. Human personnel moving in the lab require the robot to stop instantly and to replan a desired route for safety reasons. Novel algorithms like Simultaneous Localization and Mapping (SLAM) based on the distance measurements of the laser rangefinders and model based visual tracking algorithms based on the high resolution images from the camera enable the robot to discover and map an unknown laboratory without preprogramming detailed knowledge about the environment and to update it, as changes occur. Initial teaching of the lab walkthroughs is realized by utilizing a visual person tracker and gesture recognition. This way a dedicated programmer becomes obsolete.

In this paper we describe, how the model based visual person tracker has been realized to enable regular lab personnel to guide the robot through the lab for automatic map generation and for teaching positions of interest to carry out a repetitive, defined monitoring task of fermentor stations.

\section{BACKGROUND}

Visual object tracking is the process of locating moving objects in video sequences in real-time, by exploiting redundant frame-to-frame context information through model-based computer vision techniques. Recent surveys cover many state-of-the-art tracking methodologies. ${ }^{6,7}$ The Open Tracking Library (OpenTL) is an approach to unify many of the state-of-the-art model based visual tracking methodologies in a single software framework. ${ }^{8,9}$

\subsection{The OpenTL Architecture}

The software architecture of OpenTL is based on the elementary concepts of every visual tracking system. A scene with more or less prior information about it is recorded by one or multiple image sensors. The prior information enables creation of a model, which at first needs to be detected and in subsequent images needs to be tracked. Figure 3 depicts the generic flow diagram for a model-based tracking application.

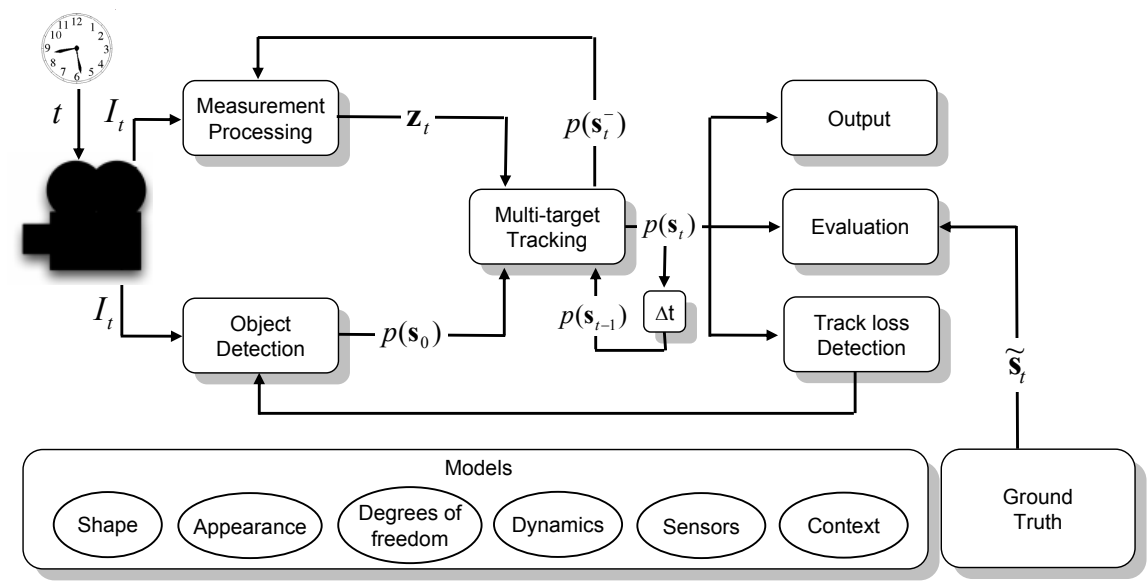

Figure 3. Generic flow diagram for a model-based visual tracking application.

\subsection{Functional Class Architecture}

The main classes that our library provides are building blocks for this kind of applications. They have been organized in functional layers representing an increasing level of abstraction: from base utilities, image acquisition devices and data structures, up to application-related functionalities (see Figure 4). 


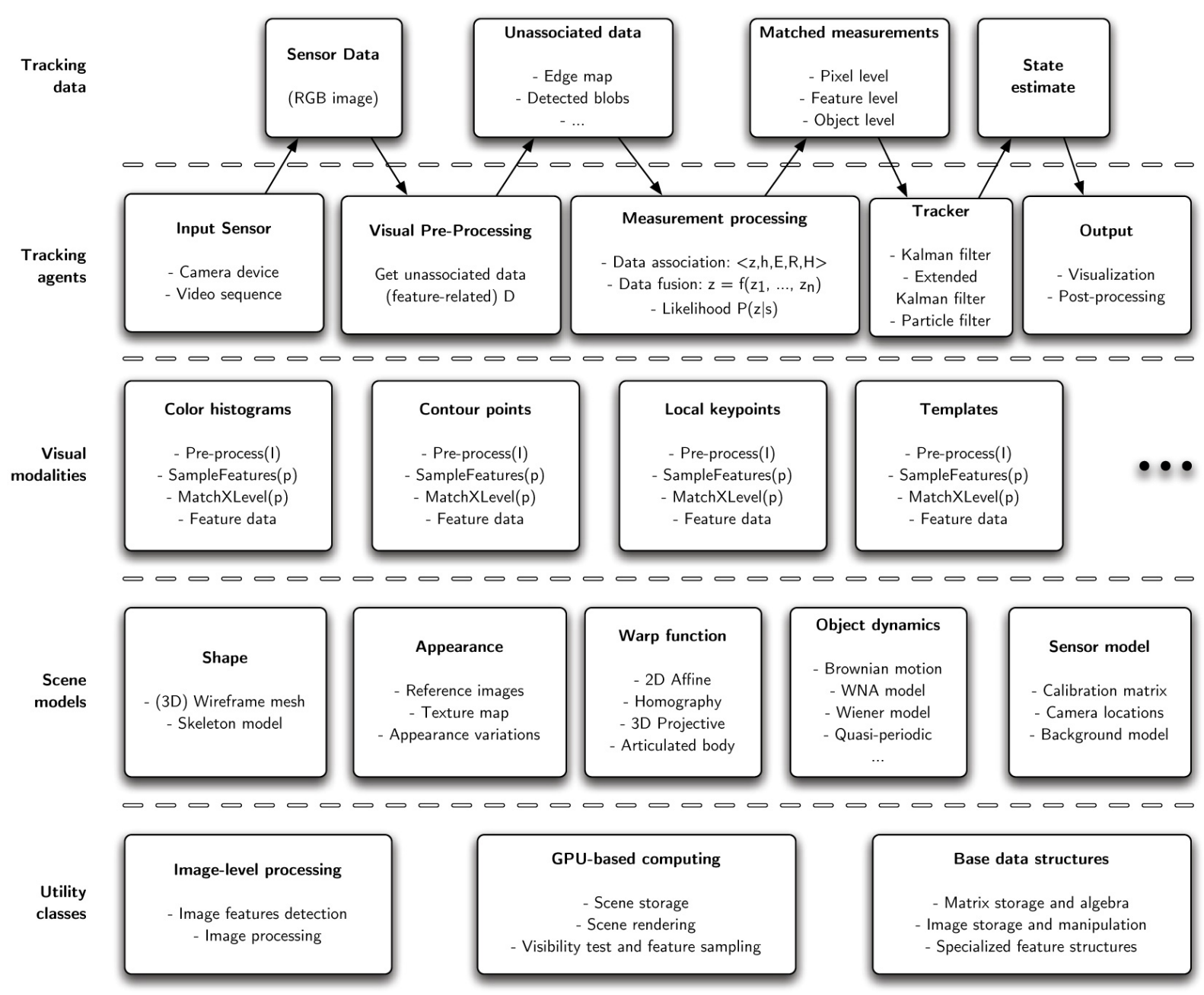

Figure 4. The layered software architecture of OpenTL with the currently implemented components.

\section{REQUIREMENTS}

The scenario in the real world Life Sciences Laboratory is as follows: lab personnel without a dedicated background shall be able to guide a mobile robotic platform through an unknown lab and to teach positions of interest, in particular fermentor stations. As the robot follows the human guide, the robot records the environment with its laser range finders to generate a map automatically via a particle filter based Simultaneous Localization and Mapping (SLAM) algorithm as described in. ${ }^{10,11}$ Based on this map the robotic platform will be able to localize itself in the lab afterwards for repetitive walkthroughs. Positions of particular interest for the monitoring task are taught by the human guide through gestures.

When starting the human guided teaching phase, the robot is required to detect the human guide and to create a multi part model based on sub-images containing the face and hands of the teacher. When the teacher moves too fast, loss-detection is required, to trigger a 'Wait for me' message. When the teacher points to a region of interest with his hand, that means, if the tracked hand is displaced horizontally from the body by a certain distance, the robot is required to record the current position as a waypoint for the repetitive walkthrough. Then, additional image processing components can detect, localize and diagnose given equipment in the lab.

This way a user friendly and robust teaching process can be realized without requiring the lab personnel to program locations of interest in a time consuming way. 


\section{IMPLEMENTATION}

The visual tracking software component for this application was designed and implemented following the layered architecture of Figure 4

\subsection{Image input}

The scene is recorded by an AVT Marlin Firewire camera, that provides RGB color images. The models in this case consist of single sub-images containing the face, the hand and the upper part of the body as well as additional reference images of known lab equipment.

\subsection{Image pre-processing}

The recorded images as well as the reference sub-images are transformed into the HSV color space to create two dimensional color histograms $z_{c o l}$ for the color-based likelihood.

\subsection{Tracker}

Each tracker holds a state-space representation of the 2D model pose, given by a planar translation $(x, y)$ and scale $h$ of the respective rectangular model in the image plane. Considering a single person tracker consisting of a hand, a head and an upper body tracker, the three particle filters provide the sequential prediction and update of the respective $2 \mathrm{D}$ states $s_{1}=\left(x_{1}, y_{1}, h_{1}\right), s_{2}=\left(x_{2}, y_{2}, h_{2}\right)$ and $s_{3}=\left(x_{3}, y_{3}, h_{3}\right)$.

Every particle filter generates several prior state hypotheses $s_{t}^{i}$ from the previous particle distribution $\left(s^{i}, w^{i}\right)_{t-1}$ through a Brownian motion model.

$$
s_{t}^{i}=s_{t-1}^{i}+v_{t}^{i}
$$

with $v$ a white Gaussian noise of pre-defined covariance in the $(x, y, h)$ state variables. A deterministic resampling strategy ${ }^{12}$ over the previous weights $w_{t-1}^{i}$ is employed every time in order to keep a good distribution of the particle set.

For each generated hypothesis, the tracker asks for a computation of the likelihood values $P\left(z_{\text {col }} \mid s_{\text {head }}^{i}\right)$ for the head, $P\left(z_{\text {col }} \mid s_{\text {hand }}^{i}\right)$ for the hand and $P\left(z_{\text {col }} \mid s_{b o d y}^{i}\right)$ for the upper body tracker.

\subsection{Color likelihood}

The rectangular reference image boxes defining the teacher's shape model are warped onto the HSV image at the predicted particle hypotheses $s_{t}^{i}$; for each patch $p$, underlying $\mathrm{H}$ and $\mathrm{S}$ color pixels are collected in the respective $2 \mathrm{D}$ histogram $q_{p}\left(s_{t}^{i}\right)$, that is compared with the reference one $q_{p}^{*}$ through the Bhattacharyya coefficient ${ }^{13}$

$$
B_{p}\left(q_{p}(s), q_{p}^{*}\right)=\left[1-\sum_{n} \sqrt{q_{p}^{*}(n) q_{p}(s, n)}\right]^{\frac{1}{2}}
$$

where the sum is performed over the $(N \times N)$ histogram bins (currently implemented with $N=10$ ).

The color likelihood is then evaluated under a Gaussian model in the overall residual

$$
P\left(z_{c o l} \mid s_{t}^{i}\right)=\exp \left(-\sum_{p} B_{p}^{2} / \lambda_{c o l}\right)
$$

with given covariance $\lambda_{\text {col }}$

\subsection{Estimated state}

Afterwards the average state $\bar{s}_{t}$

$$
\bar{s}_{t}=\sum_{i} w_{t}^{i} s_{t}^{i}
$$

is computed for each tracker.

In order to ensure jitter-free operations the trackers' outputs are finally filtered by a temporal low-pass filter with higher cut-off frequencies with respect to the expected covariance of motion parameters. 


\subsection{Loss detection}

An important feature of our system is the loss-detection, as a face or a hand disappears when the teacher turns around to lead the robot and to re-initialize the trackers as the teacher faces the robot and points at locations of interest.

In principle, target losses can be detected by checking the likelihood and covariance for the particle set. A covariance test is independent of the actual likelihood value, but it may fail to detect a loss when the particle set concentrates on a false positive which has a low covariance as well. On the other hand, the likelihood test is dependent on the likelihood value, which can vary under changing illumination conditions, however the changes would occur relatively slow considering the large amount of frames in a certain period of time.

Therefore we implemented a likelihood test on the estimated state $\bar{s}_{t}$ of each tracker and declare a loss whenever $P\left(z_{\text {col }} \mid \bar{s}_{t}\right)$ decreases below a minimum threshold value $P_{\text {min }}$. This threshold is set as a percentage (e.g. $\leq 10 \%$ ) of a reference value $P_{r e f}$, initially set to the maximum likelihood value.

In order to provide adaptivity to variable postures as well as light or shading variations, $P_{\text {ref }}$ is being slowly adapted if the last likelihood $P\left(z_{\text {col }} \mid \bar{s}_{t-1}\right)$ is comparable to $P_{\text {ref }}$ (e.g. $\geq 60 \%$ ). When a track loss occurs, the affected particle filter are re-initialized with the diffuse prior, until the target becomes visible again and the likelihood increases above the threshold.

\subsection{Action States}

By evaluating the losses and re-detections of the desired targets, the robot can distinguish between the following possible cases and take the appropriate actions:

1. teacher out of sight: don't do anything

2. teacher facing the robot: wait for 3 . or 4 .

3. teacher facing the robot and pointing at an object of interest: identify, localize and map object

4. teacher not facing the robot: follow teacher as he moves

\section{RESULTS}

Based on the previously described method we implemented a multi target tracking system for a Teaching Process to guide a mobile robot through an unknown laboratory and to teach positions of interest for subsequent, repetitive walkthroughs. Figure 5 shows the tracking system in action. When following the human teacher, the robot is learning the surrounding environment with its laser range finders for automatic map generation and for localization within this map. Figure 6 shows the map, that the robot learned in the real life sciences laboratory.

\section{CONCLUSIONS}

Autonomous vehicles became popular during the DARPA racing challenges, however mobile platforms equipped with a manipulator for increased flexibility also have been used successfully in biotech laboratories for sample management as shown on the well-known ESACT meetings. ${ }^{14}$ In this paper we have shown, how modern electronic image processing techniques on sequences of images can also be used to improve the user friendliness and versatility of a mobile robot. By the utilization of a mounted machine vision camera and visual tracking algorithms, the robot can be introduced to completely unknown locations without requiring the user to program detailed knowledge about the environment into the robot system.

\section{ACKNOWLEDGMENTS}

This work is funded by the German Ministry of Education and Research and Bayer HealthCare LLC. 

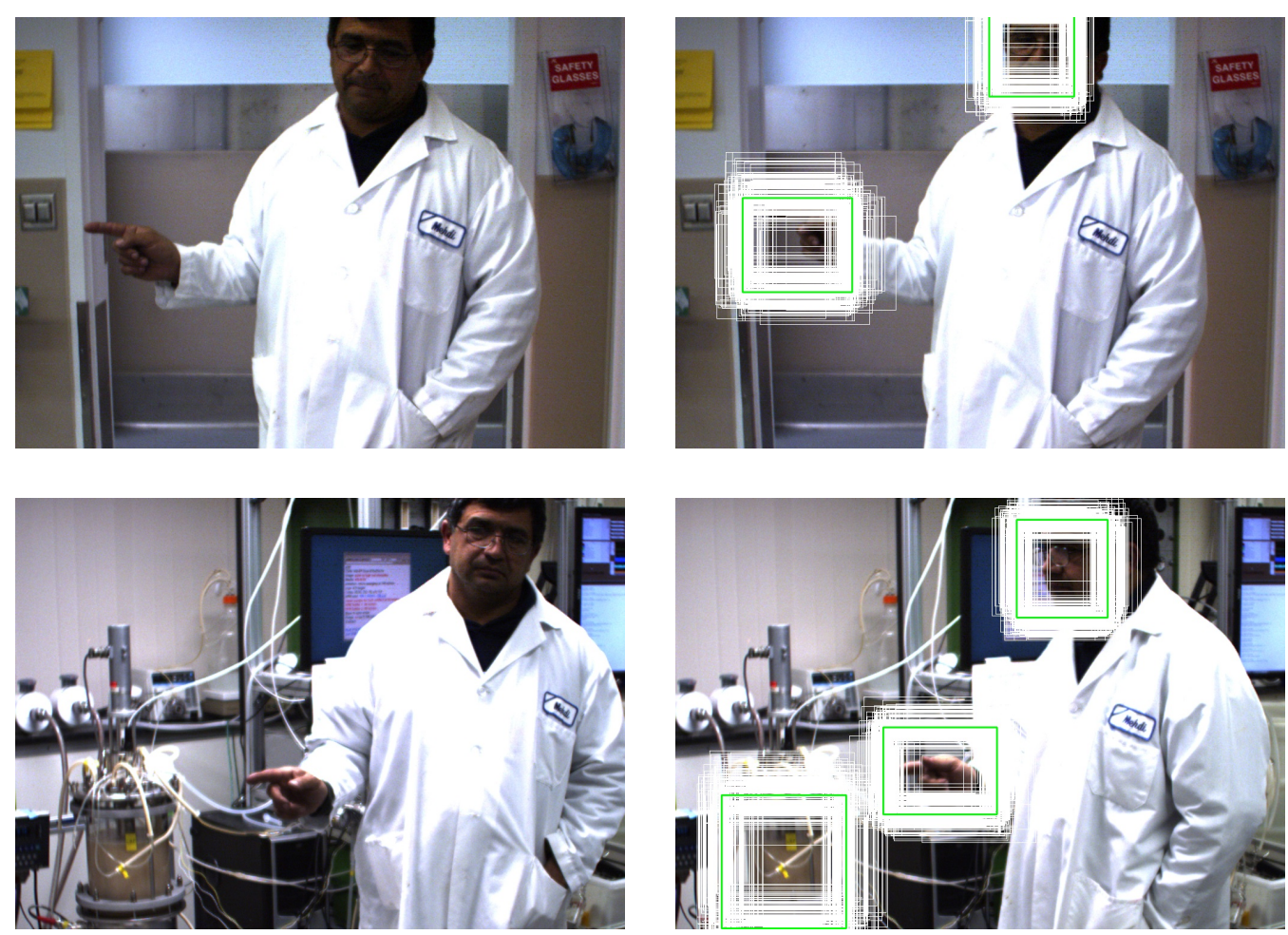

Figure 5. The multi target person tracker in action. The upper images show the face and hand tracker, while the lower images also show an identified fermentor that the scientist is pointing at. The images on the right depict the activated trackers and show the evaluated hypotheses as white rectangles and the estimated positions of the tracked targets as green rectangles.

\section{REFERENCES}

[1] Wojtczyk, M., Marszalek, M., Heidemann, R., Joeris, K., Zhang, C., Burnett, M., Monica, T., and Knoll, A., "Automation of the complete sample management in a biotech laboratory," in [Proceedings of Visions of Computer Science, BCS International Academic Conference], Abramsky, S., Gelenbe, E., and Sassone, V., eds., 87-97, The British Computer Society, Imperial College, London, UK (Sept. 2008).

[2] Lütkemeyer, D., Poggendorf, I., Scherer, T., Zhang, J., Knoll, A., and Lehmann, J., "First steps in robot automation of sampling and sample management during cultivation of mammalian cells in pilot scale," Biotechnology Progress 16(5), 822-828 (2000).

[3] Knoll, A., Scherer, T., Poggendorf, I., Lütkemeyer, D., and Lehmann, J., "Flexible Automation of Cell Culture and Tissue Engineering Tasks," Biotechnology Progress 20(6), 1825-1835 (2004).

[4] Poggendorf, I., Einsatz eines Serviceroboters zur Automatisierung der Probenentnahme und des Probenmangements während Kultivierungen tierischer Zellen in einer Technikumsumgebung, dissertation, Universität Bielefeld (June 2004).

[5] Scherer, T., A Mobile Service Robot for Automisation of Sample Taking and Sample Management in a Biotechnological Pilot Laboratory, dissertation, University of Bielefeld (Oct. 2004).

[6] Yilmaz, A., Javed, O., and Shah, M., "Object tracking: A survey," ACM Comput. Surv. 38(4), 13 (2006).

[7] Lepetit, V. and Fua, P., [Monocular Model-based 3d Tracking of Rigid Objects (Foundations and Trends in Computer Graphics and Vision(R))], Now Publishers Inc (2005).

[8] Panin, G., Lenz, C., Wojtczyk, M., Nair, S., Roth, E., Friedelhuber, T., and Knoll, A., "A unifying software architecture for model-based visual tracking," in [Image Processing: Machine Vision Applications. Edited by Niel, Kurt S.; Fofi, David. Proceedings of the SPIE, Volume 6813, pp. 681303-681303-14 (2008).], Presented at the Society of Photo-Optical Instrumentation Engineers (SPIE) Conference 6813 (Mar. 2008). 


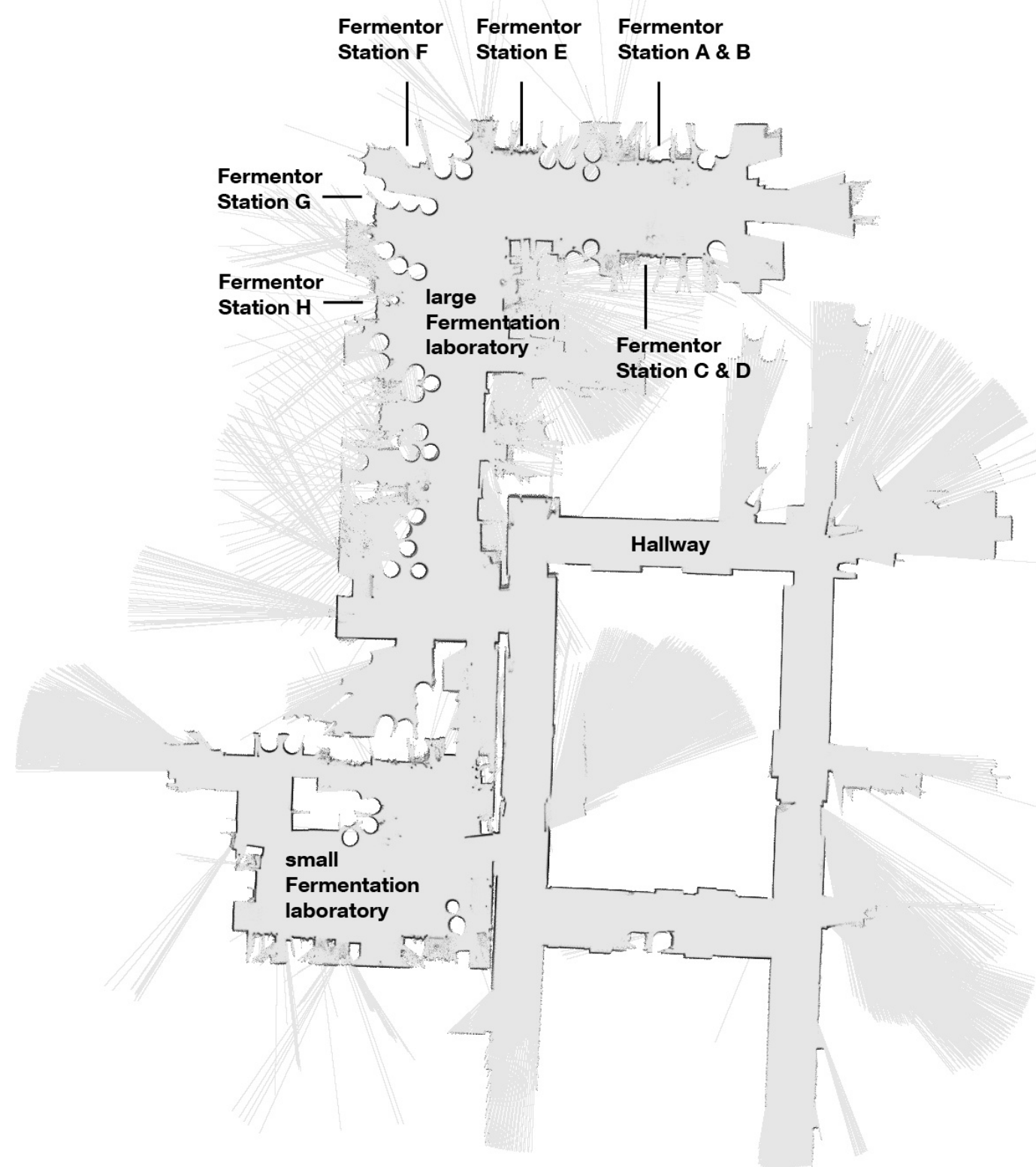

Figure 6. The automatically generated map. When following the teacher, the mobile robot records its environment with the laser range finders and automatically generates a map to be able to localize itself and to keep track of important waypoints. This figure shows the resulting map of the real biotech laboratory, where the robot is being installed and tested. 
[9] Robotics and Embedded Systems, Faculty of Informatics, Technische Universität München, Open Tracking Library (OpenTL) - A general-purpose tracking library. http://www.opentl.org/

[10] Eliazar, A. I. and Parr, R., "DP-SLAM: Fast, Robust Simultaneous Localization and Mapping Without Predetermined Landmarks," in [Proceedings of the Eighteenth International Joint Conference on Artificial Intelligence (IJCAI 03).], (2003). http://www.cs.duke.edu/ parr/ijcai03.ps.gz.

[11] Eliazar, A. I. and Parr, R., "DP-SLAM 2.0," in [IEEE 2004 International Conference on Robotics and Automation (ICRA 2004).], (2004). http://www.cs.duke.edu/ parr/dpslam2.pdf.

[12] Isard, M. and Blake, A., "Condensation - conditional density propagation for visual tracking," International Journal of Computer Vision (IJCV) 29(1), 5-28 (1998).

[13] Pérez, P., Hue, C., Vermaak, J., and Gangnet, M., "Color-based probabilistic tracking," in [ECCV '02: Proceedings of the "7th European Conference on Computer Vision-Part I], 661-675, Springer-Verlag, London, UK (2002).

[14] Wojtczyk, M., Heidemann, R., Joeris, K., Zhang, C., Burnett, M., Knoll, A., and Konstantinov, K., "The Use of a Mobile Robot for Complete Sample Management in a Cell Culture Pilot Plant," in [Cell Technology for Cell Products, Proceedings of the 19th ESACT Meeting, Harrogate, UK, June 5-8, 2005], Smith, R., ed., 543-547, Springer Netherlands (2007). 